\title{
Control Químico de la Antracnosis Causado por Colletotrichum gloeosporioides en el Cultivo del Camu Camu (Myrciaria dubia Myrtaceae) en Ucayali, Perú
}

\author{
Diana Perez ${ }^{1}$ \& José Iannacone ${ }^{2}$ \\ ${ }^{1}$ Instituto de Investigaciones de la Amazonía Peruana, IIAP, Ucayali Jr. Progreso 102, Pucallpa, Ucayali, Perú, \\ e-mail: dperez@iiap.org.pe; '2Laboratorio de Ecofisiología Animal, Facultad de Ciencias Naturales y Matemáticas, \\ Universidad Nacional Federico Villarreal, Calle San Marcos 383, Pueblo Libre, Lima, Perú, \\ e-mail: joseiannacone@gmail.com
}

(Aceptado para publicación en 01/08/2006)

Autor para correspondencia: José Iannacone

\begin{abstract}
Chemical control of anthracnose caused by Colletotrichum gloeosporioides in Camu Camu (Myrciaria dubia Myrtaceae) in Ucayali, Peru

In a plantation of camu camu Myrciaria dubia H.B.K. Mc Vaugh (Myrtaceae) in Yarinacocha city, Ucayali, Peru, the efficiency of fungicides on the control of anthracnose caused by Colletotrichum gloeosporioides (Penz.) Penz. \& Sacc. was evaluated. In general, the effect of fungicides on percentage of infection by $C$. gloeosporioides in five maturation categories for camu camu fruit was similar.
\end{abstract}

El hongo filamentoso fitopatógeno Colletotrichum gloeosporioides (Penz.) Penz. \& Sacc. es el agente causal de la antracnosis en varias especies de plantas, entre ellas Myrciaria dubia (Peraza-Sanchez et al., Journal Agricultural Food Chemistry 53:2429. 2005). El objetivo del presente trabajo fue evaluar el efecto de tres combinaciones de fungicidas: $\mathrm{T}_{1}=$ Tebuconazole-triadimenol, clorotalonil; $\mathrm{T}_{2}=$ sulfato-nitrato-fosfato de cobre; $\mathrm{T}_{3}=$ clorotalonil, sulfato-nitrato- fosfato de cobre en el control de C. gloeosporioides en el cultivo del Camu Camu en Ucayali, Perú. Los ensayos de campo se realizaron entre Junio y Agosto del 2004, en parcelas de productores de camu camu en los caseríos 7 de Junio ( $\left.08^{\circ} 17^{\prime} \mathrm{LS}, 7^{\circ} 37^{\prime} \mathrm{LW}\right)$ y Padre Bernardo $\left(30^{\circ} 10 \mathrm{LS}, 61^{\circ} 30^{\prime} \mathrm{LW}\right)$, Ucayali, Perú. Se emplearon los siguientes tres fungicidas en las siguientes dosis: $2 \mathrm{~mL}$ de Clorotalonil $\mathrm{L}^{-1}$ (Bravo ${ }^{\circledR}$, Syngenta Crop Protection, Inc. Farmex S.A.), 0,5 $\mathrm{mL}$ de Tebuconazole y Triadimenol $\mathrm{L}^{-1}$ (Silvacur Combi ${ }^{\circledR}$, Bayer Cropscience) y $3,5 \mathrm{~mL}$ de Sulfato de cobre-Nitrato de cobre-Fosfato de Cobre L ${ }^{-1}$ (Kobres ${ }^{\circledR}$, Comercial Andina Industrial S.A.). Se incluyó un testigo, el cual se trató con agua. En general, el efecto del control con fungicidas sobre el porcentaje de infección de $C$. gloeosporioides en las cinco categorías de maduración de frutos es semejante en los cuatro tratamientos (Tabla 1). Sin embargo, cuando se analizaron los resultados de cada tratramiento, se observó que el $\mathrm{T}_{3}$, fue más efectivo en cuatro categorías de frutos de camu camu: en fruto verde (índice 1), fruto verde (índice 2), fruto verde y fruto maduro. En cambio $\mathrm{T}_{1}$, fue más efectivo en fruto verde, fruto pintón y fruto maduro. El $\mathrm{T}_{2}$ en ninguna de las categorías de frutos mostró efectividad (Tabla 1). Se ha registrado alta efectividad del clorotalonil sobre la germinación de conidios, sobre la severidad e incidencia de $C$. gloeosporioides en cultivos in vitro aislados de Allium cepa L. (Haddad et al., Fitopatol. Bras. 28:435. 2003). El clorotalonil resultó ser el segundo en disminuir la severidad y el primero en disminuir la incidencia de frutos enfermos de C. gloeosporioides en Carica papaya (Papaya) en un estudio comparativo con otros siete fungicidas (Solano \& Arauz, Agronomico Costarricense 19:25. 1995). Nuestros resultados muestran que los dos tratamientos más eficientes sobre C. gloeosporioides presentaron en su composición al clorotalonil $\left(\mathrm{T}_{1} \mathrm{y}_{3}\right)$. La baja efectividad global de los tratamientos puede estar influenciada por los intervalos de aplicación que fueron heterogéneos. La alta incidencia de la enfermedad causada por C. gloeosporioides indica que el control químico con fungicidas aisladamente no es una medida eficiente para el control de la antracnosis en camu camu, señalando la necesidad de investigar otras estrategias de control (Kososki et al., Fitopatol. Bras. 26:662. 2001).

TABLA 1 - Efecto de fungicidas en el porcentajes de infección en fruto de camu camu por Colletotrichum gloeosporioides en Pucallpa, Perú

\begin{tabular}{cccccc}
\hline \hline \multicolumn{7}{c}{$\%$} \\
\hline Tratamientos & FVI1 & FVI2 & FV & FP & FM \\
\hline $\mathbf{T}_{\mathbf{0}}$ & $8,8 \pm 3,2 \mathrm{a}$ & $14,1 \pm 4,8 \mathrm{a}$ & $19,5 \pm 12,6 \mathrm{a}$ & $27,0 \pm 13,3 \mathrm{a}$ & $40,8 \pm 25,5 \mathrm{a}$ \\
$\mathbf{T}_{\mathbf{1}}$ & $8,3 \pm 4,6 \mathrm{a}$ & $11,4 \pm 5,2 \mathrm{a}$ & $12,0 \pm 5,6 \mathrm{a}$ & $9,3 \pm 5,5 \mathrm{a}$ & $25,8 \pm 12,2 \mathrm{a}$ \\
$\mathbf{T}_{\mathbf{2}}$ & $13,9 \pm 5,7 \mathrm{a}$ & $14,1 \pm 9,6 \mathrm{a}$ & $19,2 \pm 11,8 \mathrm{a}$ & $22,0 \pm 14,6 \mathrm{a}$ & $35,6 \pm 17,3 \mathrm{a}$ \\
$\mathbf{T}_{\mathbf{3}}$ & $6,4 \pm 5,4 \mathrm{a}$ & $5,2 \pm 4,3 \mathrm{a}$ & $9,5 \pm 5,5 \mathrm{a}$ & $15,9 \pm 8,0 \mathrm{a}$ & $28,5 \pm 17,1 \mathrm{a}$
\end{tabular}

Promedios seguidos por la misma letra en la columna no presentan diferencias estadísticas significativas, según la prueba de Tukey $(\mathrm{P}<0,05)$. To (Testigo). FVI1 $=$ Fruto Verde Índice $1 . \mathrm{FVI} 2=$ Fruto Verde Índice $2 . \mathrm{FV}=$ Fruto verde. FP= Fruto Pinton. FM = Fruto maduro. 